\title{
Development of artificial articular cartilage
}

\author{
BISWAJIT BERA \\ Department of Mechanical Engineering, National Institute of Technology (NIT), \\ Durgapur 713209 \\ e-mail: Bisu_bera@yahoo.com
}

MS received 20 October 2008; revised 28 April 2009

\begin{abstract}
The present study describes the development of artificial articular cartilage on the basis of mimicking structural gel properties and mechanical gel properties of natural articular cartilage. It is synthesized from PVA/Si nanocomposite containing 20\% Tetra ethoxy silane (TEOS) by sol-gel method. Mechanical strength of Poly(vinyl alcohol), PVA is improved up to $35 \mathrm{MPa}$. Manufacturing method is adopted considering colloidal stability of nano silica particle in PVA sol at specific $\mathrm{pH}=1$. An adhesive is also prepared from PVA/Si nanocomposite containing $40 \%$ TEOS for firm attachment of artificial articular cartilage on underlying bone with high bond strength.
\end{abstract}

Keywords. Artificial articular cartilage; PVA/Si nanocomposite; colloidal stability; mechanical strength; adhesive.

\section{Introduction}

The load transmitting structural members are the bones, the ends of which are sufficiently blunt or globular to provide some form of bearing area. The bone surfaces are covered with a layer of relatively soft and porous articular cartilage within the joint, and this represents the bearing material as shown in figure 1 . The joint is sealed from the surrounding tissues by the synovial membrane and the cavity thus formed is filled with synovial fluid. Indeed, nature may have discovered, in its combination of cartilage and synovial fluid, a system which is very slippery under conditions of boundary lubrication. Generally, osteoarthritis of synovial joint occurs due to gradual loss of articular cartilage during human locomotion. Till now, as suitable biomaterial to be used as an artificial articular cartilage is not available, there is need of total joint replacement surgery of osteoarthritis patient. Total joint prosthesis is commonly made with a metal component articulating against a component of ultrahigh molecular weight polyethylene (to be referred to as UHMWPE). Ultra-high molecular weight polyethylene (UHMWPE) has been choice of orthopaedic bearing material in total joint replacement surgery since 1962, but wear of UHMWPE remains major problem facing the long term success and survival of the artificial joint. One of the main reasons of failure of the artificial joint fixation into the host bone is cellular reaction against the wear debris of UHMWPE, which causes the bone resorption around the implant called osteolysis (Revell et al 1997). On the otherhand, the use of polymeric hydrogels as biomaterials is rapidly gaining importance due to their 


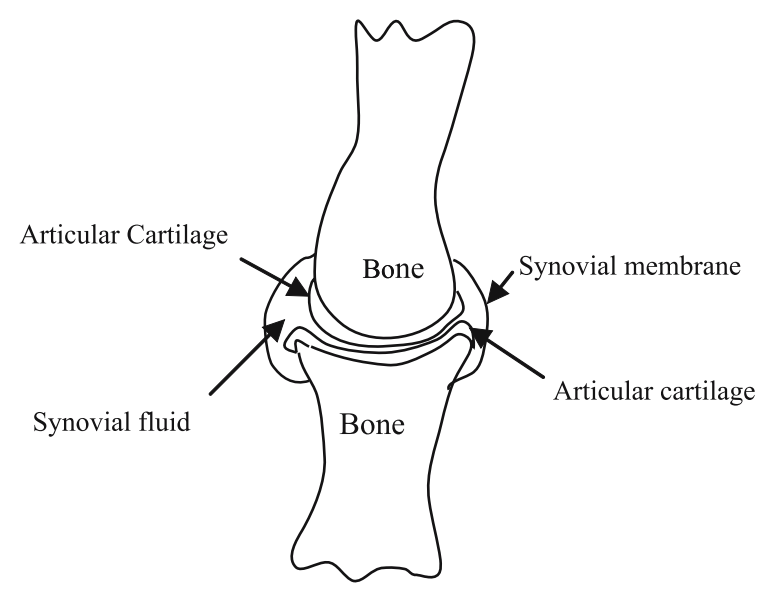

Figure 1. Simplified joint.

unique and interesting properties. The crosslinked structure present in hydrogels allows the retention of substantial amounts of water without dissolution of the polymer itself like natural tissue containing water. Nearly, 20 years ago it was anticipated that PVA hydrogel might be suitable material for artificial articular cartilage (Oka et al 1990). Though PVA hydrogel is good biocompatible material (Oka et al 2000, Kobayashi et al 2003), still it is not used due to its very limited longevity in vivo as it is having very poor ultimate strength and modulus of elasticity with respect to those of natural articular cartilage. It happens due to low strength of dry PVA itself and its strength falls down abruptly after becoming hydrogel having high degree swelling of water around 75-85\% due to low modulus of elasticity. Mechanical strength of PVA hydrogel is $0 \cdot 1-2 \cdot 8 \mathrm{MPa}$ (Bray et al 1973). There are conventional way to improve mechanical strength of PVA hydrogel by freezing and thawing method increasing hydrogen bond interaction between water and the molecular sheet of the PVA crystallite crosslink points. The mechanical strength of PVA hydrogel by freezing and thawing is increased up to $10 \mathrm{MPa}$ reported by Sasada et al (1985).

Mechanical strength of PVA hydrogel could be improved by formation of nanocomposite material. Nanocomposite hydrogel of PVA and nano HA as new artificial articular cartilage material is developed by Xu Fenglan et al but improvement of strength is not compared and reported (Fenglan Xu et al 2004). In this study, artificial articular cartilage is developed by formation of PVA/Si nanocomposite hydrogel.

\section{Sol-gel sythesis of PVA/Si nanocomposite}

\subsection{Chemical reaction}

Hydrolysis:

$$
\mathrm{Si}\left(\mathrm{OC}_{2} \mathrm{H}_{5}\right)_{4}+4 \mathrm{H}_{2} \mathrm{O} \stackrel{\mathrm{HCL}}{\Rightarrow} \mathrm{Si}(\mathrm{OH})_{4}+4 \mathrm{C}_{2} \mathrm{H}_{5} \mathrm{OH}
$$

Condensation:

$$
\begin{aligned}
& \mathrm{Si}(\mathrm{OH})_{4}+\mathrm{Si}(\mathrm{OH})_{4} \Rightarrow(\mathrm{OH})_{3}-\mathrm{Si}-\mathrm{O}-\mathrm{Si}-(\mathrm{OH})_{3}+\mathrm{H}_{2} \mathrm{O} \\
& \left\{(\mathrm{OH})_{3}-\mathrm{Si}-\mathrm{OH}+\mathrm{H} \mathrm{O}-\mathrm{Si}-(\mathrm{OH})_{3}\right\}
\end{aligned}
$$


Polycondensation

$$
\left(-\stackrel{\mid}{S} i-O-{ }_{\mid}^{\mid} i-\right)_{n} .
$$

A 10\% dilute alcoholic sol of PVA was prepared by dissolving $10 \mathrm{~g}$ PVA in $100 \mathrm{ml}$ boiling distilled water. After complete dissolution, the Poly(vinylalcohol) sol is cooled at room temperature. Concentrated Hydro Chloric Acid (HCL) was used as the acidic catalyst for the formation of nanosized spheroidal silica particle from alkoxysilane, TEOS. A sufficient amount of concentrated HCL was added gradually, to attain $\mathrm{pH}=1$ of the alcoholic PVA sol. Subsequently, required weight percentage of Tetra Ethoxysilane (TEOS) with respect to $100 \mathrm{~g}$ PVA was added into the PVA alcoholic sol, and, the mixture were agitated by a magnetic stirrer for $45 \mathrm{~min}$ at room temperature to synthesize colloidal monodispersed nanosilica in situ PVA alcoholic sol through fast condensation reaction. Thereafter, colloidal PVA/Si nanocomposite sol was cast in Petri dish and finally, it was dried in hot air oven at $50^{\circ} \mathrm{C}$ to make thin film for sample preparation.

\section{Mechanism of PVA/Si nanocomposite formation}

Stober et al (1968) synthesized the silica particle in base medium. Similar method was followed for synthesis of silica particle in acid medium. In this regard, Uragami et al (2002) have also synthesized the silica particle in situ PVA alcoholic sol but mechanism is not reported in detail.

\subsection{Synthesis of nanosilica particle}

Figure 2 schematically illustrates the process of nucleation and subsequent growth of silica particle in situ Poly(vinyl alcohol) sol. When the concentration of alkoxy silane (TEOS) increases as a function of time due to mechanical agitation by magnetic stirrer, no nucleation of silica particle would occur even above the equilibrium solubility $\left(\mathrm{C}_{\mathrm{eq}}\right)$. The nucleation of silica particle occurs through hydrolysis and condensation reaction only when the supersaturation reaches a certain value $\left(\mathrm{C}_{\mathrm{nu}}\right)$ above the solubility, which corresponds to the energy barrier

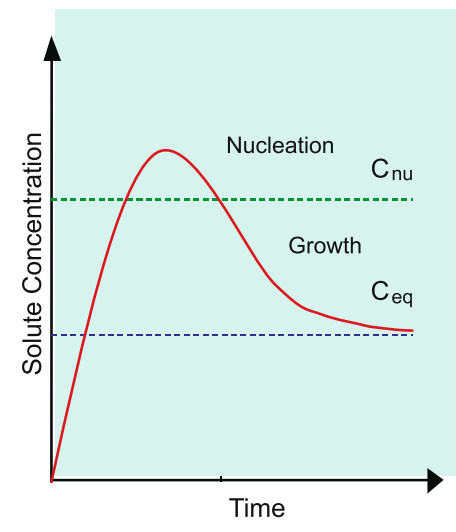

Figure 2. Nucleation and growth. 
$\left[\left.\right|_{H^{+}}\right.$
Figure 3. Coordination bond with $H^{*}$ ion.

for formation of nuclei. After the initial nucleation of silica particle, the concentration of growth species decreases and the change of Gibbs free energy reduces. When concentration decreases below the specific concentration, which corresponds to the critical energy, no more nuclei of silica particle would form, whereas the growth will proceed until the concentration of growth species has attained the equilibrium concentration or solubility.

\subsection{Colloidal stability of nanosilica particle}

Whenever one insoluble phase is in contact with another liquid phase, an ionic distribution occurs at the interface of the two phase. After formation of silica particle, $\mathrm{H}^{+}$ion will penetrate into the pores of silica gel and like metal ligand, the silica particle will form coordination bond with $\mathrm{H}^{+}$by transferring a lone pair of oxygen as shown in figure 3 . Also, $\mathrm{H}^{+}$ions will be absorbed to a certain extent onto the surface of each silica particle. This will render the surface negatively charged which would naturally drag some negative ions $\left(\mathrm{OH}^{-} \mathrm{or} \mathrm{Cl}^{-}\right)$in its vicinity. At equilibrium, electrical double layer will exist at the particle-liquid interface. One part of the double layer with positive charge on the fixed surface of each silica particle and second part of the double layer with negative charge in the liquid phase as shown in figure 4 . The second part of double layer negatively charged comprises two parts-a fixed part and a diffuse portion with concentration of negative charge gradually falling off. The total potential drop from solid surface to solution is called double layer potential and the potential difference between two fixed charge surface is termed as zeta potential. Thus, HCL apparently influence the shape and size of created silica particles. As $\mathrm{pH}$ in acid medium increases with increment of HCL concentration, stable size of very small silica particle (even nano scale) will be formed in situ PVA alcoholic medium.

\subsection{Structure of PVA/Si nanocomposite}

Figure 5 depicts the PVA/Silica network. During PVA sol preparation, pendent hydroxyl group of PVA polymeric chain will form inter hydrogen bonding with water molecule of the sol by breaking intra hydrogen bonding. To form gel of colloidal silica in situ PVA sol, polymeric chains will be physically crosslinked by intra hydrogen bonding of hydroxyl groups through evaporation of water molecule after crossing the gel point. After gentle drying of the PVA/Si gel in hot air oven, free water molecule attached with hydroxyl group of the 


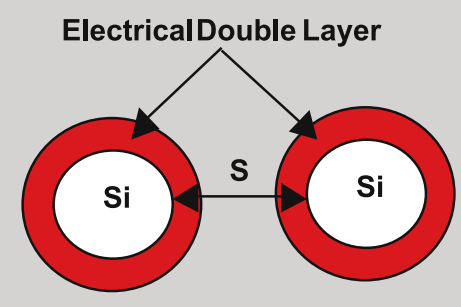

No Overlap-No Repulsion

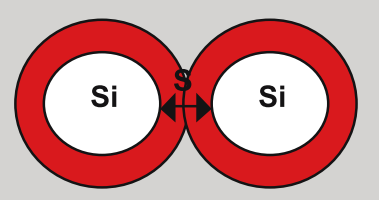

Overlap -Repulsion
Figure 4. Colloidal stability of silica particle.

nanocomposite gel will evaporate. Consequently, free hydroxyl group of silica will be attached with pendent hydroxyl group of Poly(vinylalcohol) gel through inter hydrogen bonding during drying. Due to rigid structure of silica, shape and size of nanosilica will not change after dehydration. During drying of the composite sol, HCL will evaporate disturbing the stability of the nanosilica particles but association of the nanoparticle by diffusion will be resisted by PVA stabilizer due to increment of viscosity caused by drying.

\section{Basis of artificial articualr cartilage development}

Artificial articular cartilage could be developed through mimicking the properties of natural articular cartilage.

\subsection{Structural gel properties}

Natural cartilage is a rigid porous collagenous network containing $75-85 \%$ water and basically, it is not swelled after absorption of water along with hyaluronic acid. Structural gel

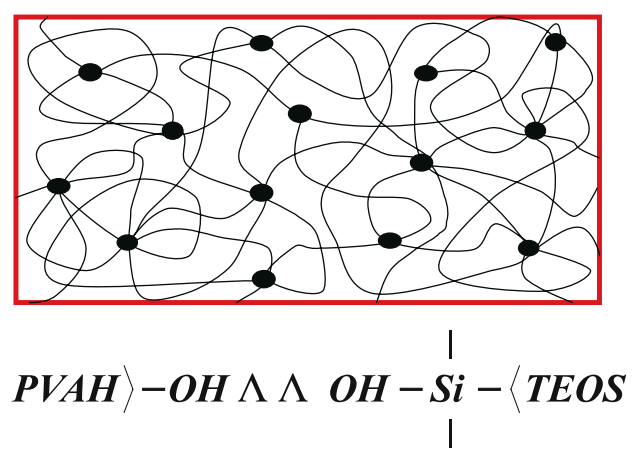

Figure 5. PVA/Nano Si network. 
properties of artificial articular cartilage were determined by study of swelling behaviour of PVA/Si nanocomposite containing 0\%, 10\%, 20\%, 30\%, 40\%, and 50\% TEOS. Among the constituents of synovial fluid, hyaluronic acid has taken only to measure the degree of swelling because other constituents like glycoprotien and phospolipid would be adsorbed on the polymer nanocomposite which would contribute error in evaluation of equilibrium degree of swelling. So, swelling in synovial fluid in vivo is equivalently considered by swelling in hyaluronic acid only in vitro. Aqueous solution of $0.40 \%$ hyaluronic acid is prepared after dissolving $0.40 \mathrm{~g}$ sodium hyaluronate salt in $100 \mathrm{ml}$ distilled water at ambient conditions. The different PVA/Si nanocomposite thin film were dried completely in hot air oven at average $50^{\circ} \mathrm{C}$ and weighted. The films were immersed into an aqueous solution of $0.40 \%$ hyaluronic acid. After equilibrium swelling of the nanocomposite films for 5 days under ambient conditions, the films were taken out and weighted after wiping out by filter paper immediately.

$$
\phi=\frac{w_{f}-w_{i}}{w_{f}} \times 100,
$$

where $w_{i}$ and $w_{f}$ are initial and final film weight respectively.

Here, swelling index, $\varphi$ is evaluated with respect to final weight of the nanocomposite gel to find out the percentage of interstitial fluid i.e. water content in the artificial articular cartilage and to compare with the percentage of interstitial fluid content (75-80\%) in natural biphasic articular cartilage. Swelling index decreases exponentially due to increment of modulus of elasticity and decrement of pores of PVA matrix as filled by nanosilica particles. Swelling index of PVA/Si nanocomposite decreases exponentially and there is no significant change of swelling index starting from 20\% TEOS content as shown in figure 6 which indicates that there is no change of shape and size of the nanocomposites. So, starting from $20 \%$ TEOS content, the nanocomposites behave like unhydrated gel due to its rigid gel structure but PVA is basically hydrated gel due to its low modulus of elasticity. So, the PVA/Si nanocomposite containing 20\% TEOS content is having critical swelling index because it determines change of structural gel property of PVA from hydrated PVA gel to unhydrated PVA gel.

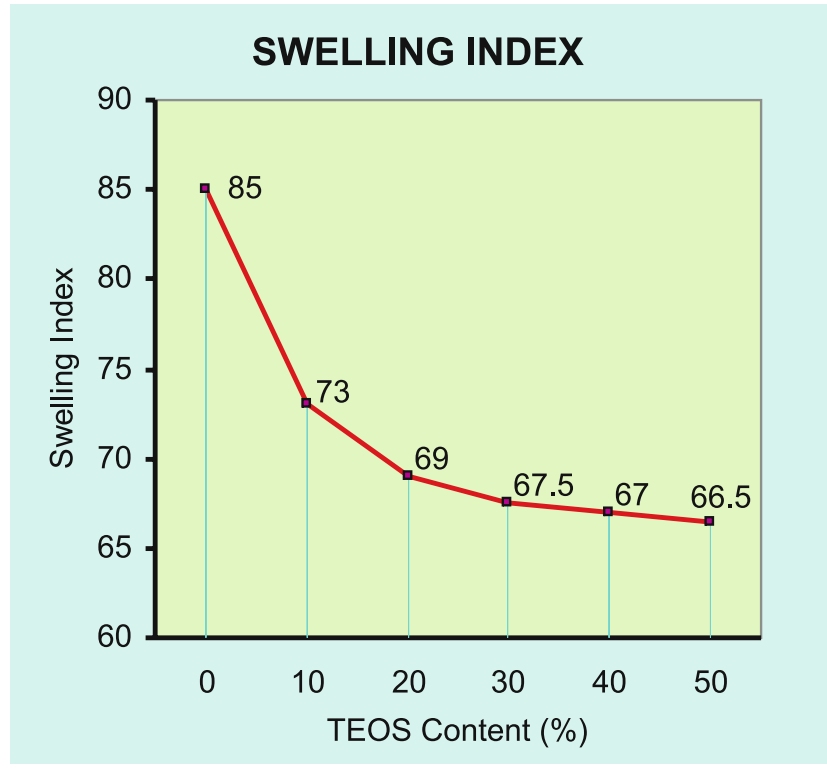

Figure 6. Swelling index. 
Starting from $20 \%$ TEOS content, PVA/Si nanocomposites is maintaining rigid gel structure mimicking gel structure of rigid collagenous network of natural cartilage but limit of mechanical property should be selected from stress-strain relationship of the nanocomoposite gel mimicking mechanical property of natural cartilage.

\subsection{Mechanical gel properties}

The mechanical properties of the nanocomposite gel film were determined by using a universal testing machine (UTM Zwick 1445) on tensile specimens punched out from the cast film using ASTM die C. Tensile stress strain curves for PVA/Si nanocomposite gels containing $20 \%, 30 \%, 40 \%$ and $50 \%$ TEOS are depicted in figure 7 .

PVA/Si nanocomposite gel including around $20 \%$ by weight TEOS having critical swelling index is considered as reference mechanical property for the unhydrated nanocomposite gels. It is observed that after around 20\% TEOS content, mechanical strength of PVA/Si nanocomposites increases, but it behaves as a brittle material. So, mimicking of ductile property of natural articulate cartilage, in that event can not be achieved. It is observed that only, the PVA/Si nanocomposites including around $20 \%$ by weight TEOS, having critical swelling index follow sufficient plastic deformation before failure and behave almost like a ductile material, thereby mimicking the property of natural cartilage. However, lower limb joints are subject to an impact load of approximately seven times of body weight during jumping or running. The artificial joint after cartilage replacement should sustain injuries in such events due to extremely high loads that are applied in very short intervals where rise of load from zero to peak value might occur within $1 \mathrm{sec}$. So, PVA/Si nanocomposite including around $20 \%$ by weight TEOS content has to be selected as artificial articular cartilage and its ultimate strength has been found to be around $35 \mathrm{MPa}$. The artificial cartilage have enough mechanical strength and damping effect for long time durability of artificial joint after cartilage replacement.

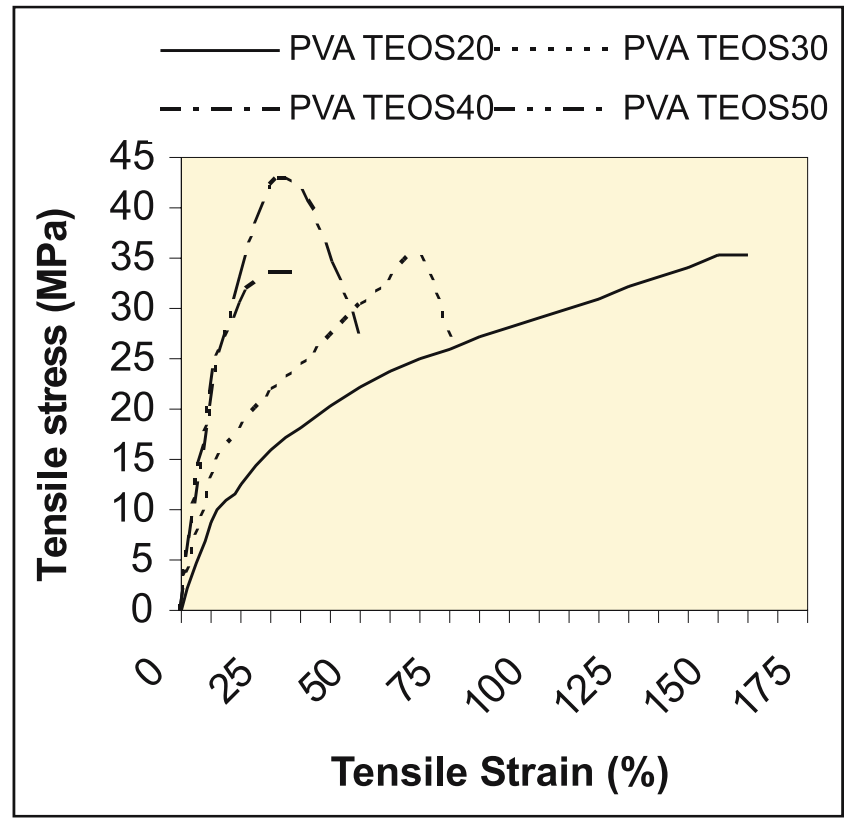

Figure 7. Stress strain diagram. 


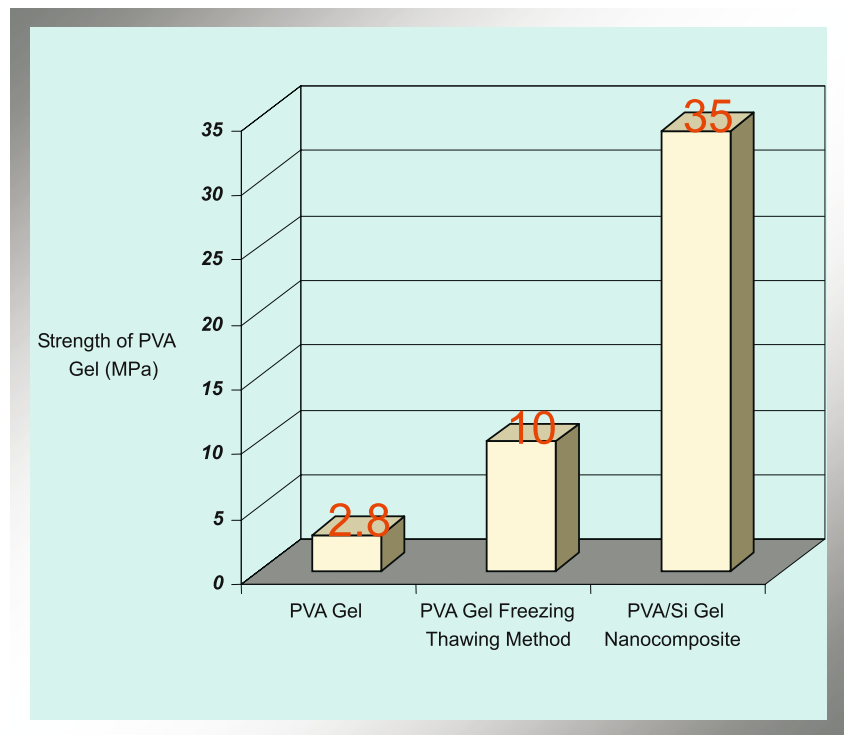

Figure 8. Comparison of mechanical strength.

From the comparison manifested in the accompanying figure 8 , it ought to be adequately clear that mechanical strength of PVA hydrogel is improved from $2.8 \mathrm{MPa}$ to $35 \mathrm{MPa}$ after formation of PVA/Si nanocomposite hydrogel containing around $20 \%$ by weight TEOS, thereby facilitating mimicking the property of natural cartilage. In this regard, it is reported that mechanical strength of PVA gel is improved up to $10 \mathrm{MPa}$ by freezing thawing method (Sasada et al 1985).

\subsection{Firm attachment to underlying bone}

Other than the long term durability of PVA hydrogel, it is very difficult to fix on underlying bone. Since as PVA-H itself does not adhere to bone, suture fixation could be performed but long term fixation of PVA-H by suture is very difficult (Kobayashi et al 2003). Natural bone is made of composite material i.e. Hydroxy Apatite (HA) ceramic material in collagenous matrix. So, silica glass ceramic material could be considered as substitute of bone material. To understand the natural adhesion property of the artificial articular cartilage on underlying bone, direct casting of PVA/Si nanocomposite is done in glass petri dish. It is observed that the artificial articular cartilage is firmly adhered on silica glass forming calcium silicate $\left(\mathrm{Ca}_{2} \mathrm{SiO}_{4}\right)$. It indicates the very good adhesion property of artificial articular cartilage to underlying bone like fixation of natural cartilage. In this regard, an adhesive is prepared with PVA/Si nanocomposite gel containing around $40 \%$ TEOS. Specifically, $40 \%$ TEOS is selected from figure 7 to produce high strength of bond in between artificial articular cartilage and underlying bone after replacement of osteoarthritic cartilage. The adhesive produces ionic bond with $\mathrm{HA}$ of bone by forming calcium silicate $\left(\mathrm{Ca}_{2} \mathrm{SiO}_{4}\right)$. It produces strong covalent bond with artificial articular cartilage forming siloxane group $(-\mathrm{Si}-\mathrm{o}-\mathrm{Si}-)$ and also produces hydrogen bond in between PVA and PVA. As the thin layer of adhesive (greater than molecular dimension) is having large percentage of nanosilica particle, it could resist high shear strength of artificial joint under high loading condition. 


\section{Conclusion}

PVA is anticipated as a suitable biomaterial to be used as artificial articular cartilage but it is not used due to poor durability in vivo. Sufficient mechanical strength of PVA is improved after formation of PVA/Si nanocomposite containing 20\% TEOS to sustain huge joint loading during hard working like running or jumping, etc. An adhesive is also developed for firm attachment of artificial articular cartilage on underlying bone so that it could not be loosen during complicated loading condition. Final conclusion is that it will bring new paradigm in joint replacement surgery of osteoarthritis patient.

\section{References}

Bray J C, Merrill E W 1973 Poly(vinyl alcohol) hydrogels for synthetic articular cartilage material. J. Biomed. Mater. Res. 7: 431-443

Fenglan Xu, Yubao Li, Xuejiang W, Jie W, Aiping Y 2004 Preparation and characterization of nanohydroxyapatite/poly(vinyl alcohol) Hydrogel biocomposite. J. Mater. Sci. 39: 5669-5672

Kobayashi M, Toguchida J, Oka M 2003 Preliminary study of polyvinyl alcohol-hydrogel (PVA-H) artificial meniscus. Biomaterials 24: 639-647

Oka M, Nouguchi T, Kumar P, Ikeuchi K, Yamamuro T, Hyon S H, Ikada Y 1990 Development of an artificial articular cartilage. Clin. Mater. 6: 361-381

Oka M, Ushio K, Kumar P, Ikeuchi K, Hyon S H, Nakamura T, Fujita H 2000 Development of artificial articular cartilage. Proc. Inst. Mech. Eng. Part-H 214: 59-68

Revell P A, AL-Saffar N, Kobayashi A 1997 Biological reaction to debris in relation to joint prostheses. Proc. Inst. Mech. Eng. - Part H 211: 187-197

Sasada T, Takahashi M, Watanabe M, Mabuchi K, Tsukamoto Y, Nanbu M 1985 Frictional behaviour of a total hip prosthesis containing artificial articular cartilage. J. Jpn. Soc. Biomaterial 3: 151-157

Stober W, Fink A, Bohn E 1968 Control growth of monodisperse silica spheres in the micron size range. J. Colloid and Interface Sci. 26: 62-69

Uragami T, Okazaki K, Masugi H, Miyata T 2002 Structure and permeation characteristics of an aqueous ethanol solution of organic-inorganic hybrid membrances composed of Poly(vinyl alcohol) and Tetraethoxysilane. Macromolecules 35: 9156-9163 